\title{
Identification of Tetraphenylborate Radiolysis Products in a Simulated Feedstock for Radioactive Waste Processing (U)
}

by

\author{
R. E. Eibling
}

Westinghouse Savannah River Company

Savannah River Site

Aiken, South Carolina 29808

M. G. Bartett

School of Chemistry and Biochemistry, Georgia Institute GA USA

R. E. Carson

School of Chemistry and Biochemistry, Georgia Institute GA USA

S. A. Tostino Jr.

School of Chemistry and Biochemistry, Georgia Institute GA USA

G. J. Kunkel

School of Chemistry and Biochemistry, Georgia Institute GA USA

R. F. Browner

School of Chemistry and Biochemistry, Georgia Institute GA USA

K L Busch

School of Chemistry and Biochemistry, Georgia Institute GA USA

A document prepared for ENVIRONMENTAL SCIENCE AND TECHNOLOGY at from ? - ?.

DOE Contract No. DE-AC09-89SR18035

This paper was prepared in connection with work done under the above contract number with the U.S.

Department of Energy. By acceptance of this paper, the publisher and/or recipient acknowledges the U.S.

Government's right to retain a nonexclusive, royalty-free license in and to any copyright covering this paper, along with the right to reproduce and to authorize others to reproduce all or part of the copyrighted paper. 


\section{DISCLAIMER}

This report was prepared as an account of work sponsored by an agency of the United States Government. Neither the United States Government nor any agency thereof, nor any of their employees, makes any warranty, express or implied, or assumes any legal liability or responsibility for the accuracy, completeness, or usefulness of any information, apparatus, product, or process disclosed, or represents that its use would not infringe privately owned rights. Reference herein to any specific commercial product, process, or service by trade name, trademark, manufacturer, or otherwise does not necessarily. constitute or imply its endorsement, recommendation, or favoring by the United States Government or any agency thereof. The views and opinions of authors expressed berein do not necessarily state or reflect those of the United States Government or any agency thereof.

This report has been reproduced directly from the best available copy.

Available to DOE and DOE contractors from the Office of Scientific and Technical Information, P. O. Box 62, Oak Ridge, TN 37831; prices available from (615) 576-8401.

Available to the public from the National Technical Information Service, U. S. Department of Commerce, 5285 Port Royal Rd., Springfield, VA 22161 


\section{DISCLAIMER}

Portions of this document may be illegible in electronic image products. Images are produced from the best available original document. 


\section{Identification of Tetraphenylborate Radiolysis Products in a}

\section{Simulated Feedstock for Radioactive Waste Processing}

Michael G. Bartlett, Richard E. Carlson, Samuel A. Testino, Jr., Gary J. Kunkel, Richard F. Browner, and Kenneth L. Busch

School of Chemistry and Biochemistry, Georgia Institute of Technology, Atlanta, Georgia, 30332-0400

Russell E. Eibling

Westinghouse Savannah River Company

Savannah River Technology Center

Aiken, South Carolina, 29808 


\begin{abstract}
Using gas chromatography/mass spectrometry, liquid secondary ion mass spectrometry, inductively coupled plasma/mass spectrometry, ion chromatography, and high performance liquid chromatography, over 50 organic and inorganic species have been identified in the aqueous portion of a simulated feedstock for tetraphenylborate hydrolysis. The major organic species present are benzene, phenol, benzamide and a variety of substituted phenylphenols. The major inorganic species present are sodium, nitrite, and oxalate ions.
\end{abstract}




\section{Introduction}

The Savannah River Site has generated about 83 million gallons of high-level radioactive aqueous waste since startup in 1954. The first step in treatment of this waste is adjustment with sodium hydroxide and evaporation of the water to reduce the overall volume to 35 million gallons. The insoluble metal oxides and hydroxides produce a precipitate known as sludge. The soluble radioactive species are removed through the addition of sodium tetraphenylborate. The tetraphenylborate addition causes the formation of a viscous white precipitate, called the precipitate slurry. The final step of the disposal process involves immobilization of the radioactive species in both the precipitate slurry and the sludge. This process, known as vitrification involves passing a current through a mixture of glass frit and waste in order to convert the mixture into a stable glass product. This glassified product can then be stored for extended periods in a secure facility.

The first step towards immobilization of the soluble radioactive species in borosilicate glass is the addition of sodium tetraphenylborate (TPB) and sodium titanate to the radioactive aqueous solution. These species cause the precipitation of radioactive cesium-137 and the adsorption of strontium-90 and plutonium. The precipitate also includes a number of inorganic and organic species, present both as trace impurities in the original mixture, and as products of radiolysis of the solution and of the precipitate. The organic portion of this precipitate, including the tetraphenylborate itself, must be removed for compatibility with the vitrification process. The intermediate steps in processing concern themselves with the organic removal process. In the presence of formic acid, the tetraphenylborate precipitate is hydrolyzed to generate benzene. Hydrolysis of the TPB is enhanced by the presence of a copper (II) catalyst. It is the mixture of organic compounds formed by radiolysis and the effect of those compounds on the hydrolysis process and on the chemistry of the copper catalyst that has been the focus of recent research efforts.

Initial studies of the tetraphenylborate hydrolysis process have found that some component of the radiolysis mixture inactivates the copper catalyst.(5) The interaction of organic materials with the catalyst, and the subsequent interference with the hydrolysis process, would have presented problems with the use of the Vitrification process as an effective means for the disposal of radioactive waste. Prevention of the catalyst deactivation is obtained by washing the irradiated tetraphenylborate precipitate in the Late Wash Facility prior to hydrolysis to remove the soluble radiolysis products. Identification of the organic radiolysis products, their distribution in the Late Wash Facility, and their interactions with the copper catalyst has become an important analytical issue.

To further investigate the reaction products of the TPB precipitation process, a simulated feedstock was created from compounds known to be present in the starting materials. This simulated feedstock was precipitated with sodium tetraphenylborate and then 
exposed to Co-60 gamma radiation to simulate two years of additional storage time (200 megarad) prior to the hydrolysis process. The irradiated product was divided into two parts, the filtered supernatant liquid and the precipitate slurry, which contains the TPB and the solid sodium titanate. This report deals with the chemical analysis of the supernatant.

To date, over fifty different compounds have been identified in the filtrate. Many of the identifications have been made using gas chromatography/mass spectrometry (GC/MS) and ion chromatography (IC), with support from liquid chromatography, and direct spectrometric analysis using secondary ion mass spectrometry.

\section{Experimental}

An ethyl acetate extract of the supernatant was prepared by taking $300 \mathrm{~mL}$ of the TPB filtrate (the sample was used as received from the site, $\mathrm{pH}$ of the filtrate was 9.3) and evaporating the solution under a stream of dry nitrogen to a volume of approximately 20 $\mathrm{mL}$. Next, the concentrated filtrate was extracted using $70 \mathrm{~mL}$ of high purity ethyl acetate (Burdick and Jackson), followed by 4 additional extractions using $50 \mathrm{~mL}$ of ethyl acetate. Finally, the fractions were combined and evaporated to approximately $0.5 \mathrm{~mL}$. The ethyl acetate extract was used for GC/MS and HPLC analysis.

GC/MS data was obtained using a Hewlett-Packard 5890 Series II gas chromatograph with a HP 5971 Series Mass Selective Detector. The gas chromatographic column was a $\mathrm{J} \& W$ Scientific DB-5 (30m $\times 0.25 \mathrm{~mm} \times 1.0 \mu)$ column. A 2 microliter injection was used with a temperature program of $100(2)-10-300(1)$. A second portion of the ethyl acetate extract was examined for non-volatile components and polar components through derivatization of the mixture with bis(trimethylsilyl)trifluoroacetamide (BSTFA) and $1 \%$ trimethylchlorosilane (TMCS). The derivatization was carried out by adding $1 \mathrm{~mL}$ of the BSTFA/TMCS mixture to $25 \mathrm{mg}$ of the ethyl acetate extract. The mixture was held at 70 degrees $\mathrm{C}$ for 15 minutes. A white precipitate formed, and was allowed to settle. The supernatant was removed, concentrated to $100 \mu \mathrm{L}$, and $1 \mu \mathrm{L}$ was injected for GC/MS analysis. A temperature program of $35(2)-20-300(2)$ was used to separate the components of the derivatized mixture.

HPLC data was obtained using a Hewlett-Packard 1090 Series II liquid chromatograph with a photodiode array detector, and HPLC Chemstation (Pascal Series) software. A 25 $\mu \mathrm{L}$ injection of the ethyl acetate extract was made on a Dupont Zorbax $25 \mathrm{~cm} \times 4.6 \mathrm{~mm}$ C-18 column. Spectral measurement was performed over a range of 190 to $600 \mathrm{~nm}$. An $\mathrm{H}_{2} \mathrm{O} / \mathrm{CH}_{3} \mathrm{CN}$ gradient elution method with a flow rate of $1.0 \mathrm{~mL} / \mathrm{min}$ was used; the initial concentration of $20 \%$ acetonitrile was increased to $100 \%$ over 25 minutes and was held until the last component eluted after 33 minutes. The UV/vis spectra of eluting peaks were compared to spectra contained in an original spectral library. The library was generated by individually analyzing several standards of compounds suspected to be present in the extract using chromatographic conditions identical to those described above, and measuring their UV/vis spectra and recording standard retention times. 
Ion chromatographic separations were completed with a Dionex DX-300 IC with a conductivity detector. An AS-12 column $(2 \mathrm{~mm} \times 25 \mathrm{~cm})$ was used to separate the anions while a CS-10 column $(2 \mathrm{~mm} \times 25 \mathrm{~cm})$ was used to separate the cations directly from a 50:1 dilution of the TPB filtrate. Anions were eluted under isocratic conditions with $20 \mathrm{mM} \mathrm{NaOH}$ (Aldrich). Cations were eluted under isocratic conditions with 20 $\mathrm{mM}$ methane sulfonic acid (Fluka). The solvent flow rate was $0.25 \mathrm{~mL}$ per minute.

Inductively coupled plasma/mass spectrometry (ICP/MS) was completed using a PerkinElmer/Sciex Elan Model 250 ICP/MS system. The samples was prepared by taking 1 $\mathrm{mL}$ of the TPB filtrate, adding $50 \mathrm{~mL}$ of concentrated nitric acid, and heating overnight at $70^{\circ} \mathrm{C}$. The acid-digested sample was concentrated under nitrogen to a volume of 1 $\mathrm{mL}$, and diluted to $50 \mathrm{~mL}$ using $18.3 \mathrm{M} \Omega$ deionized water. The sample was then introduced into the plasma and quantitated using the method of standard addition. Argon flow rates for the plasma, carrier gas, and auxiliary gas were $14,0.95$ and $1.2 \mathrm{~L} / \mathrm{min}$ respectively. The sampling height for the mass analyzer was $18 \mathrm{~mm}$, and the plasma power was $1.2 \mathrm{~kW}$ with a reflected power of less than 5 watts.

Secondary ion mass spectrometry was performed on a VG-70SEQ hybrid mass spectrometer with $\mathrm{EBqQ}$ geometry. Samples were ionized using a $\mathrm{Cs}^{+}$ion gun operated at $30 \mathrm{keV}$. Ions were extracted from the source at $8000 \mathrm{eV}$. The TPB filtrate was added directly to the probe tip and the solvent evaporated using a heat gun. No matrix was used for the analysis.

\section{Results and Discussion}

A large number of compounds found in the TPB filtrate have been identified through the combined use of GC/MS and HPLC. Table 1 contains the complete list of identified compounds, and the techniques that provided confirmation for each compound. A typical GC total ion trace is shown in Figure 1. Note that the mass selective detector was turned off during the elution of phenol (identified in a previous run) to prevent it from overloading the detector. Phenol, the three phenylphenol isomers, and the benzene were identified (as shown in Figure 1) through spectral and retention time matches. There are many more eluting peaks resolved in the GC trace than have been positively identified. In most cases, a positive identification is difficult due to the similar chromatographic and mass spectral properties of positional isomers, and the unavailability of standards.

Initial work involving the TPB filtrate involved extraction using cyclohexane. However, cyclohexane was found to be relatively poor in extraction of the organic components compared to ethyl acetate. This comparison was made by carrying out the extraction of the TPB filtrate using both cyclohexane and ethyl acetate under identical conditions, with GC/MS evaluation of the extracted components. Ethyl acetate was used as the extraction solvent of choice then for most GC/MS work. Derivatization of the mixture present in the ethyl acetate extract was carried out to provide data for the more polar components of the mixture. A trimethylsiloxy derivatization reaction was completed, and, as shown in 
Figure 2, many more resolved peaks are now visible in the GC/MS trace. The most significant new compounds that have been identified using derivatization are the benzenediols and the hydroxybenzonitriles. The mass spectrum of 1,3bis(trimethylsiloxy)benzene, along with the NBS library standard spectrum, are in shown in Figure 3. The spectra show a match of $94 \%$ using a reverse search algorithm. The interest in the benzene diols and the organic nitriles stems from the possibility of these organic compounds coordinating with a copper ion in solution, and changing the catalytic activity.

Ion chromatography (IC) has been used to identify the many cationic and anionic species that are found in high $(0.001$ to $0.1 \mathrm{M})$ concentrations in the TPB filtrate. The identities of the anions are of particular importance because of the chance that an insoluble copper salt could be formed when the solubility product is exceeded. Figure 4 shows the IC separation achieved for anions from the TPB filtrate. The identity of each ion is shown above the corresponding peak; these were established by matching the retention times. Quantitation was completed by preparing calibration curves for each of the ions identified through retention time matches. The ionic composition of the TPB filtrate, along with determined concentrations, are shown in Table 2 . The presence of carbonate cannot be conclusively determined because the TPB filtrate solution absorbs carbon dioxide from the atmosphere and will be a function of storage conditions and sample handling procedures. The formation of insoluble copper salts appears to be a significant concern in these mixtures since copper formate will be added to create a solution that is $950 \mathrm{ppm}$ in copper. The greatest concern centers around the formation of copper oxalate which has a $\mathrm{K}_{\mathrm{sp}}$ of $1.6 \times 10^{-19}$. The $\mathrm{pH}$ of the filtrate solution is 9.7 , which can generate an additional potential problem in formation of copper (II) hydroxide $\left(\mathrm{K}_{\mathrm{sp}}\right.$ of $4.43 \times 10^{-10}$ ) if sufficient formic acid is not added.(6) According to the Savannah River Site, formic acid is added to hydrolyze the tetraphenylborate. The formic acid is added to decrease the $\mathrm{pH}$ to a level where hydrolysis can occur. However, work by Gupta and co-workers has shown that the $\mathrm{pH}$ of the TPB filtrate will increase during radiolysis.(3) This increase in hydroxide concentration could cause the copper ions to form copper (II) hydroxide, thus decreasing the catalytic activity.

Cation analysis of the TPB filtrate using ion chromatography revealed only two species, sodium and ammonium ions. The concentration of each species is shown in Table 2 . It is noteworthy that the total concentration of the cations present in the TPB feedstock is nearly as the total concentration of anions (excluding carbonate) present in the sample.

Inductively coupled plasma/mass spectrometry has been used to identify and quantitate the transition metals present in the TPB filtrate. Originally, we surveyed for 31 elements. Of the 31 metals analyzed, four transition metals were found to be present at the $\mathrm{ppb}$ level. Their identities and concentrations are summarized in Table 3. The presence of titanium was expected since it is used (as insoluble sodium titanate) in the adsorption of radioactive strontium. The lack of dissolution of the sodium titanate is shown by the very low concentration of the titanium in the supernatant liquid. The presence of the chromium copper, and zinc are attributed to leaching from the containers. 
The TPB filtrate feedstock has not had the copper (II) catalyst added, so this is not a viable source of copper.

Direct secondary ion mass spectrometric analysis of the supernatant mixture has revealed the presence of sodium borate, a compound that has not been identified by any other means. The ion corresponding to this compound has been identified through exact mass measurement as $\left[\mathrm{HBO}_{3} \mathrm{Na}_{3}\right]$, indicating the presence of sodium borate.

\section{Conclusion}

Presently, solubility is being targeted as the major problem with the copper catalyst when it is added in the tetraphenylborate hydrolysis process. The high concentration of oxalate ions present in the irradiated TPB filtrate solution could prevent copper from being an effective catalyst. However, the addition of a final washing process in the Late Wash Facility to remove soluble species produced by radiolysis will prevent the oxalate ion interference. There is a need to evaluate the efficiency of the acid hydrolysis process with respect to $\mathrm{pH}$ changes related to radiolysis.

If the organic compounds listed in Table 1 continue to remain a concern, then the two classes that should be targeted for closer inspection are the biphenols and the benzenediols. We have speculated that the hydroxyl groups on these compounds may be able to coordinate the copper ions in solution. The present approach focuses on determination of the extent of interaction of these species with the copper ion. The paramagnetic nature of copper excludes the use of nuclear magnetic resonance spectroscopy to determine the interaction of the metal with these organic hydroxides. The use of UV-vis spectroscopy to seek absorption shifts due to metal-oxygen interactions are now being investigated.(7)

\section{References}

1. "Processing of Tetraphenylborate Precipitates in the Savannah River Site Defense Waste Processing Facility," WSRC-90-MS-150.

2. "DWPF Technical Basis for HAN Precipitate Hydrolysis Process," WSRC-TM90-11.

3. Gupta, A. K; Hanarahan, R. J.; and Walker, D. D. J. Phys. Chem., 1991, 95, 3590 .

4. Gupta, A. K; Hanarahan, R. J.; and Walker, D. D. Radiochim. Acta, 1993, $\underline{60}, 43$.

5. "Mass Spectroscopic Studies on Tetraphenylborate Chemistry" SRL-WERP-920114. 
6. The Handbook of Chemistry and Physics, 67th Ed., 1987, CRC Press, Boca Raton, B-207.

7. Yamamoto, Y.; Okamoto, N.; and Tao, E. Anal. Chim. Acta, 1969, 47, 127. 


\section{Table 1.}

Organic species identified from the tetraphenylborate filtrate solution and the technique use for the identification.

\section{Compound Name}

1. Phenol

2. 2-Phenylphenol

3. 3-Phenylphenol

4. 4-Phenylphenol

5. Benzene

6. Aniline

7. 2-Phenoxyphenol

8. 3-Phenoxyphenol

9. Biphenyl

10. 2,2'-Biphenol

11. 4,4'-Biphenol

12. Benzamide

13. Cyclohexane

14. 1,1'-Biphenol-3-amine

15. Dimethylmercury

16. $1,1^{\prime}: 4^{\prime}, 1^{\prime \prime}$-Terphenyl

17. Bicyclohexane

18. Triphenylborane

19. Boric Acid

20. Benzyl Alcohol

21. Benzoic Acid

22. 2-Hydroxybenzonitrile

23. 3-Hydroxybenzonitrile

24. 4-Hydroxybenzonitrile

25. 1,2-Benzenediol

26. 1,3-Benzenediol

27. 1,4-Benzenediol

28. 2-Nitrophenol

29 3-Nitrophenol

30. 1,2,3-Benzenetriol

31. Phenylazophenol

\section{GC/MS TMS Derivative HPLC}

$\begin{array}{lll}X & X & X \\ X & X & X \\ X & X & X \\ X & X & X\end{array}$

$\mathrm{X}$

$\mathrm{X}$

$\mathrm{X}$

$\mathrm{X}$

$\mathrm{X}$

X

$\mathrm{X}$

X

$\mathrm{X}$

$\mathrm{X}$

$X$

$\mathrm{X}$

$\mathrm{X}$

$\mathrm{X}$
X

$\mathrm{X}$

$X$
$X$
X

$\mathrm{X}$

X

$\mathrm{X}$

X

$\mathrm{X}$

$X$

$X$

X

$\mathrm{X}$

X

X

$\mathrm{X}$

$X$

$\mathrm{X}$

$\mathrm{X}$

$\mathrm{X}$ 
Table 2.

Ion identities and concentrations as determined by ion chromatography.

\section{Ion Name $\quad$ Concentration (M)}

$\begin{array}{lll}\text { 1. } & \text { Fluoride } & 0.0093 \\ \text { 2. } & \text { Acetate } & 0.034 \\ \text { 3. } & \text { Formate } & 0.0099 \\ \text { 4. } & \text { Carbonate } & 0.20 \\ \text { 5. } & \text { Chloride } & 0.037 \\ 6 . & \text { Nitrite } & 0.023 \\ 7 . & \text { Sulfate } & 0.0012 \\ 8 & \text { Oxalate } & 0.0061 \\ 9 . & \text { Nitrate } & 0.00057 \\ 10 . & \text { Sodium } & 0.12 \\ 11 . & \text { Ammonium } & 0.0081\end{array}$

Table 3.

Metal identities and concentrations as determined by inductively coupled plasma/mass spectrometry.

Metal Name

Chromium

Copper

Titanium

Zinc
Concentration (ppb)

41

23

162

14 


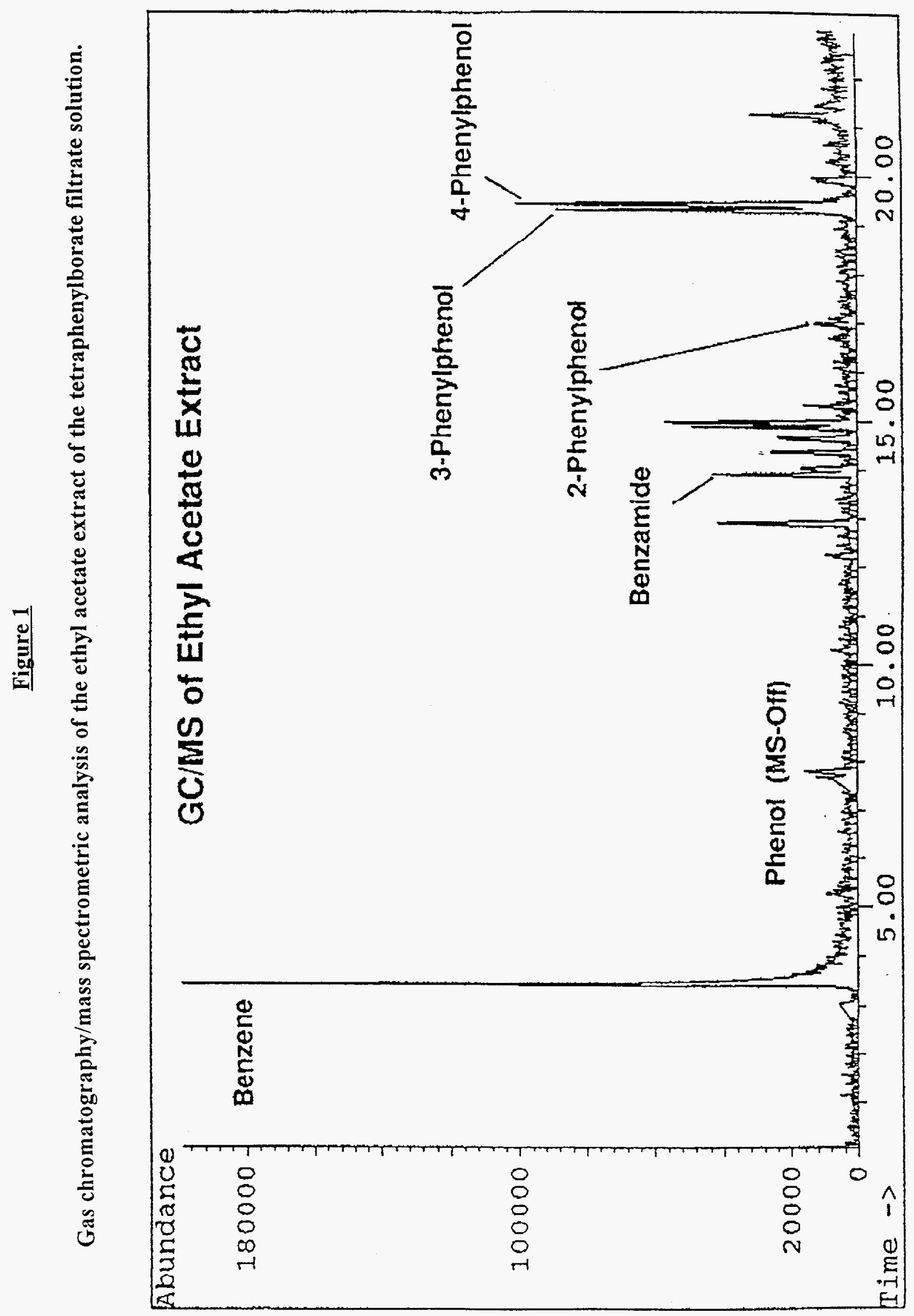


Gas chromatography/mass spectrometric analysis of the TMS derivative of an ethyl acetate extract of the tetraphenylborate filtrate solution.

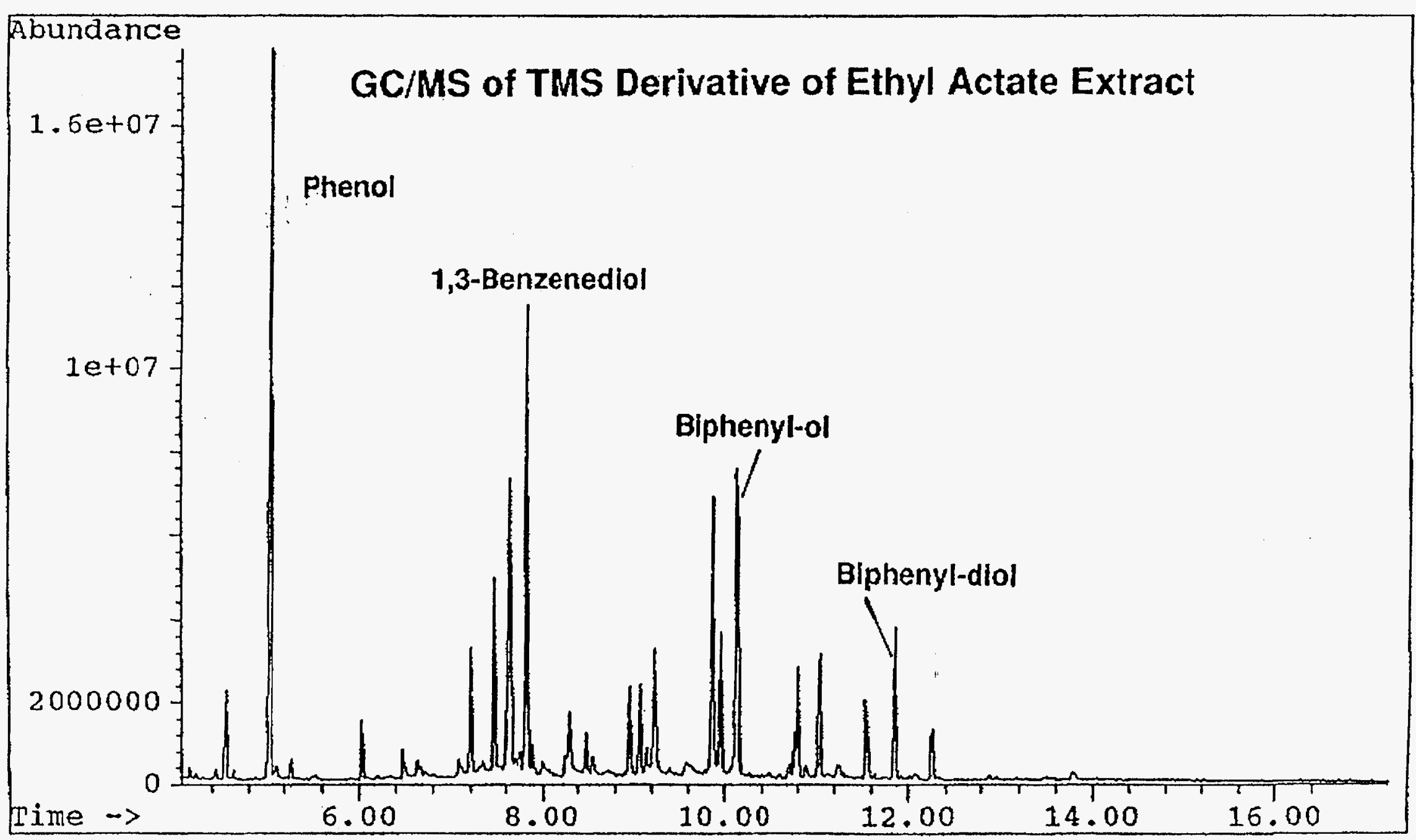




\section{Figure 3}

Comparison of the mass spectrum of a peak from the TMS derivative of the ethyl acetate extract to a library mass spectrum of 1,3-Bis(trimethylsiloxy)benzene.

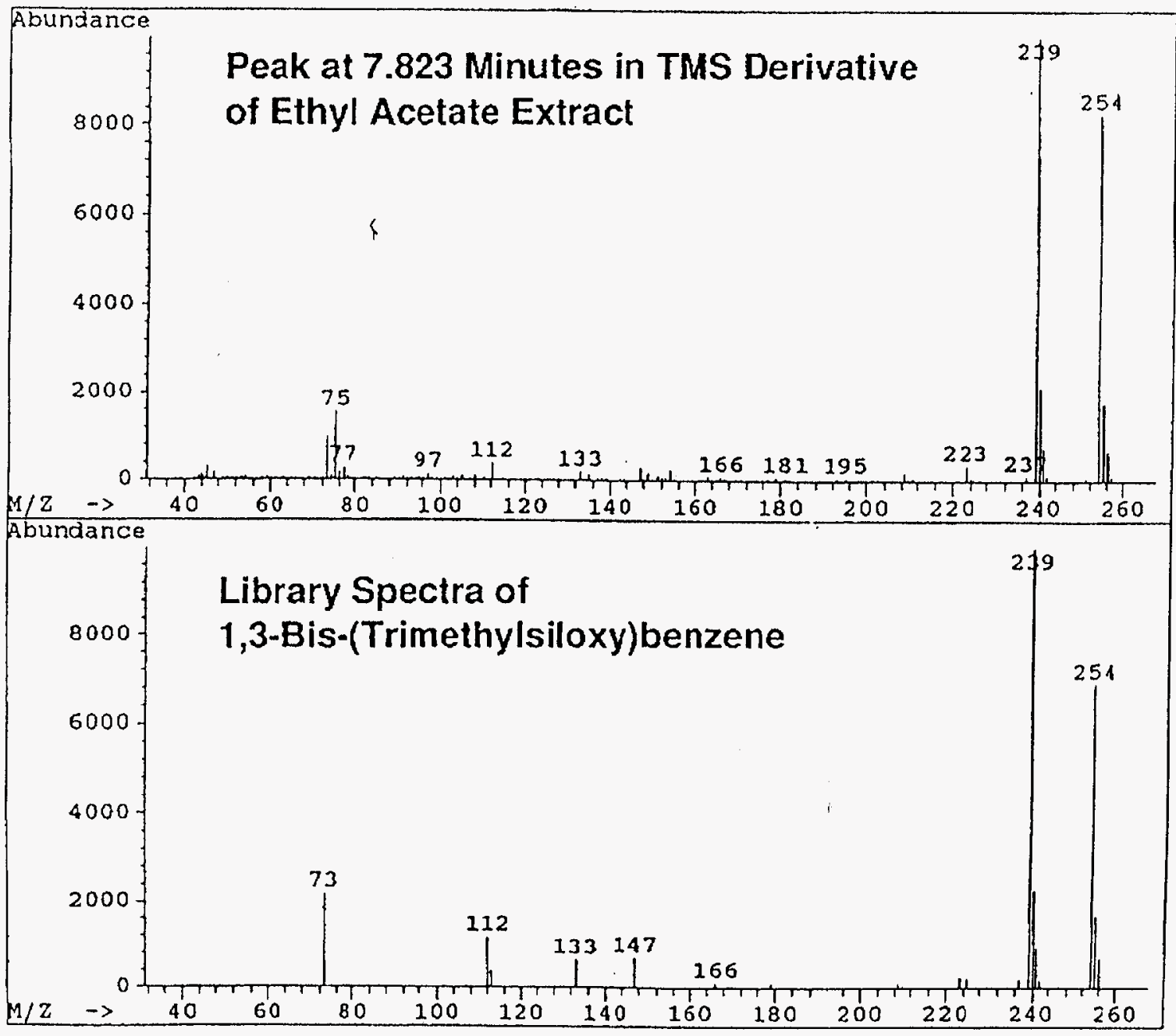




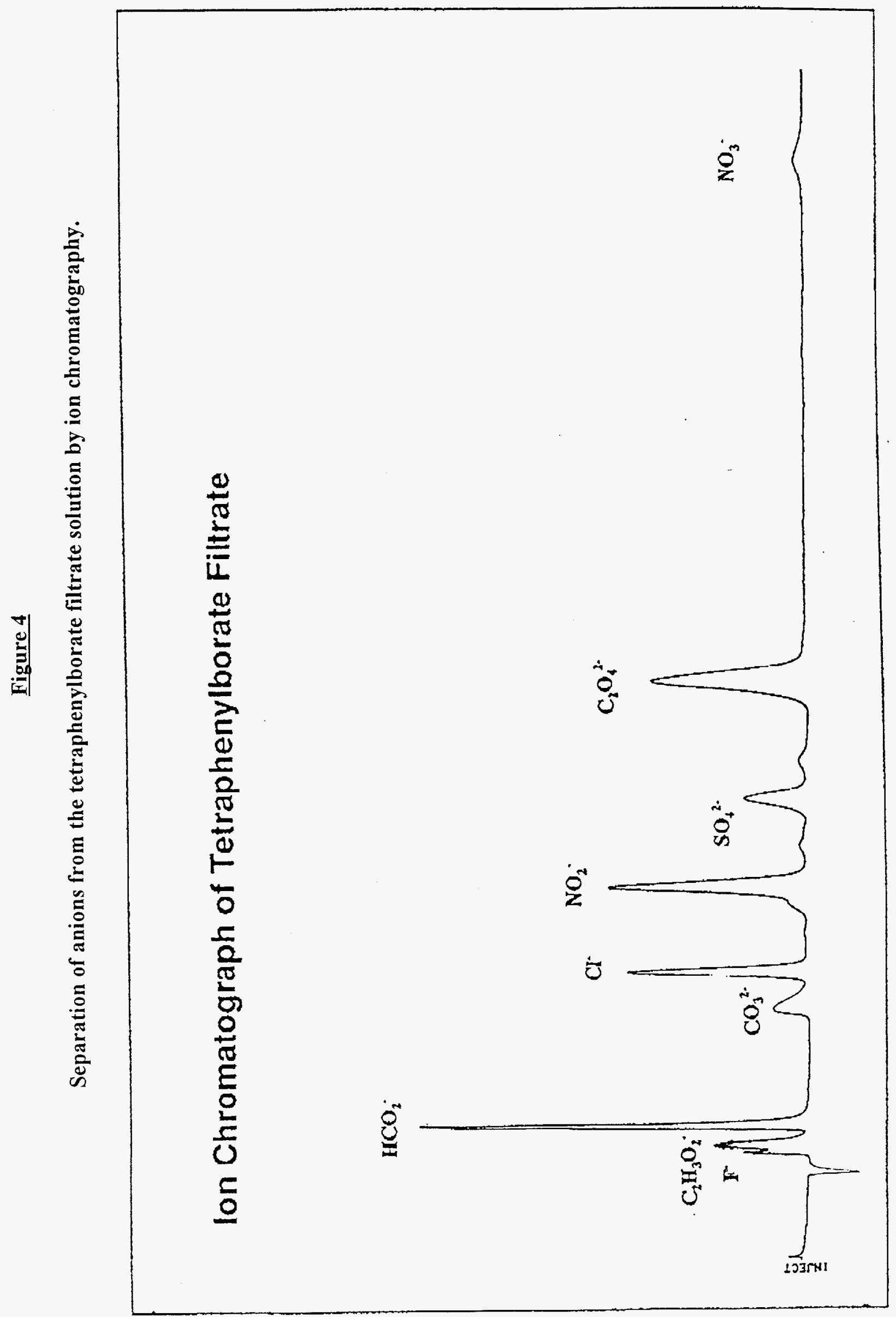

